\title{
Benign Recurrent Intrahepatic Cholestasis Type 1 with Novel Nonsense Mutations in the ATP8B1 Gene
}

\author{
Ryoichi Miura ${ }^{a}$ Tomokazu Kawaoka $^{a}$ Michio Imamura ${ }^{a}$

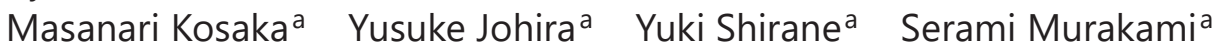 \\ Shigeki Yano ${ }^{a}$ Kei Amioka ${ }^{a}$ Kensuke Naruto $^{a}$ Yuwa Ando $^{a}$ \\ Yumi Kosaka $^{a}$ Kenichiro Kodama ${ }^{a}$ Shinsuke Uchikawa ${ }^{a}$ Hatsue Fujino ${ }^{a}$ \\ Atsushi Ono $^{a}$ Takashi Nakahara $^{a}$ Eisuke Murakami ${ }^{a}$ Masami Yamauchi ${ }^{a}$ \\ Takao Hinoi $^{b}$ Hiroshi Aikata ${ }^{a}$ \\ aDepartment of Gastroenterology and Metabolism, Graduate School of Biomedical and \\ Health Science, Hiroshima University Hospital, Hiroshima, Japan; bepartment of Clinical \\ and Molecular Genetics, Hiroshima University Hospital, Hiroshima, Japan
}

\section{Keywords}

ATP8B1 - Autosomal recessive liver disease $\cdot$ Benign recurrent intrahepatic cholestasis

\begin{abstract}
Benign recurrent intrahepatic cholestasis (BRIC) is a group of genetically heterogeneous autosomal recessive liver disorders characterized by recurrent episodes of jaundice and pruritus. BRIC is divided into two groups, BRIC type 1 (BRIC1) and BRIC type 2 (BRIC2), caused by mutations in the ATP8B1 and $A B C B 11$ genes. We show that novel nonsense mutations in ATP8B1 (c.2989G >A, C.1547T>A) are the cause of BRIC1. A 16-year-old girl presented with severe jaundice. Acute and chronic liver diseases with infectious (hepatitis virus), metabolic, and autoimmune etiologies were excluded. Imaging revealed normal intra- and extra-hepatic bile ducts. Liver biopsy revealed severe intrahepatic bile stasis with bile plugs. She had similar symptoms at the age of 0 years. The BRIC criteria were satisfied, and ATP8B1 and ABCB11 gene analyses performed. Surprisingly, novel nonsense variants of the ATP8B1 gene (c.2989G $>$ A and c.1547T>A) in heterozygosis were found, which were identified in each of her parents. Therefore, the compound heterozygote was thought to cause BRIC1 in these patients. Genetic mutations that differ from those already known may help diagnose patients with BRIC.
\end{abstract}




\section{Introduction}

Benign recurrent intrahepatic cholestasis (BRIC) is a group of genetically heterogeneous autosomal recessive diseases, characterized by recurrent episodes of cholestatic jaundice that resolves spontaneously within a few weeks to a few months. The first case of BRIC was described by Summerskill and Walshe in 1956 [1]. The disease can occur at any age and is associated with recurrent episodes of pruritus and increase in serum bilirubin and bile acid (BA) without elevation of serum gamma-glutamyltransferase (GGT). BRIC1 is associated with mutations in the ATP8B1 gene (chromosome 18q21-22), which encodes a protein called familial intrahepatic cholestasis 1 (FIC1). In this study, we report a case of BRIC1 in a young woman who was diagnosed based on the clinical history, biochemical and histological features; diagnosis was confirmed by genetic analyses, which we have at the best of knowledge, first provided the evidence of compound heterozygous and novel mutations in the ATP8B1 gene (c.2989G>A, c.1547T>A).

\section{Case Report}

A 6-month-old girl presented at a general hospital on account of diarrhea followed by jaundice and pruritus of several days duration. Laboratory investigations showed total bilirubin (T-Bil) levels of $17.3 \mathrm{mg} / \mathrm{dL}$ (conjugated fraction: $13.5 \mathrm{mg} / \mathrm{dL}$ ), serum BA levels of $304 \mu \mathrm{mol} / \mathrm{L}$, and mildly elevated alkaline phosphatase; aspartate aminotransferase, alanine aminotransferase and GGT were within normal limits (Table 1). Abdominal ultrasonography and computed tomography revealed neither enlargement of the liver nor mass in the liver. The intra- and extra-hepatic biliary tree and pancreatic ductal system showed no obstruction or dilation. Hepatobiliary scintigraphy showed excretion of bile through the ducts, although it was slightly delayed. Liver biopsy showed severe intrahepatic bile stasis with bile plugs; no damage to the hepatocytes and bile ducts was observed. Because genetic cholestasis was suspected, administration of urusodeoxycholic acid, colestyramine, and phenobarbital led to an improvement in symptoms. Since then, she had no episodes of

Table 1. Laboratory findings on admission at 0 years and 6 months

\begin{tabular}{llll}
\hline Hematology & \multicolumn{3}{c}{ Biochemistry } \\
WBC & $9,000 / \mu \mathrm{L}$ & AST & $50 \mathrm{IU} / \mathrm{L}$ \\
RBC & $396 \times 104 / \mu \mathrm{L}$ & ALT & $47 \mathrm{IU} / \mathrm{L}$ \\
$\mathrm{Hb}$ & $10.9 \mathrm{~g} / \mathrm{dL}$ & $\mathrm{LDH}$ & $214 \mathrm{IU} / \mathrm{L}$ \\
$\mathrm{PLT}$ & $43.0 \times 104 / \mu \mathrm{L}$ & ALP & $1740 \mathrm{IU} / \mathrm{L}$ \\
Biochemistry & & GGT & $17 \mathrm{IU} / \mathrm{L}$ \\
$\mathrm{TP}$ & $5.2 \mathrm{~g} / \mathrm{dL}$ & BUN & $9 \mathrm{mg} / \mathrm{dL}$ \\
Alb & $3.3 \mathrm{~g} / \mathrm{dL}$ & $\mathrm{Cr}$ & $0.3 \mathrm{mg} / \mathrm{dL}$ \\
T-Bil & $17.3 \mathrm{mg} / \mathrm{dL}$ & BA & $304 \mu \mathrm{mol} / \mathrm{L}$ \\
D-Bil & $13.5 \mathrm{mg} / \mathrm{dL}$ & & \\
\hline
\end{tabular}

WBC, white blood cell; RBC, red blood cell; Hb, hemoglobin; Plt, platelet; TP, total protein; Alb, albumin; T-Bil, total bilirubin; D-Bil, direct bilirubin; AST, aspartate aminotransferase; ALT, alanine aminotransferase; LDH, lactate dehydrogenase; ALP, alkaline phosphatase; GGT, gamma glutamyl transpeptidase; BUN, blood urea nitrogen; $\mathrm{Cr}$, creatinine; BA, bile acid. 
Table 2. Laboratory findings on admission at 16 years

\begin{tabular}{llll}
\hline Hematology & \multicolumn{3}{c}{ Biochemistry } \\
WBC & $6.34 / \mu \mathrm{L}$ & $\mathrm{TP}$ & $6.7 \mathrm{~g} / \mathrm{dL}$ \\
Neut & $73.10 \%$ & Alb & $3.2 \mathrm{~g} / \mathrm{dL}$ \\
$\mathrm{RBC}$ & $434 \times 104 / \mu \mathrm{L}$ & $\mathrm{T}-\mathrm{Bil}$ & $1.3 \mathrm{mg} / \mathrm{dL}$ \\
$\mathrm{Hb}$ & $11.9 \mathrm{~g} / \mathrm{dL}$ & $\mathrm{AST}$ & $47 \mathrm{IU} / \mathrm{L}$ \\
PLT & $51.3 \times 104 / \mu \mathrm{L}$ & $\mathrm{ALT}$ & $56 \mathrm{IU} / \mathrm{L}$ \\
& & $\mathrm{LDH}$ & $326 \mathrm{IU} / \mathrm{L}$ \\
Coagulation & & $\mathrm{ALP}$ & $1319 \mathrm{IU} / \mathrm{L}$ \\
PT & $92 \%$ & $\mathrm{GGT}$ & $27 \mathrm{IU} / \mathrm{L}$ \\
PT-INR & 1.01 & $\mathrm{BUN}$ & $12.7 \mathrm{mg} / \mathrm{dL}$ \\
APTT & $29.9 \mathrm{~s}$ & $\mathrm{Cr}$ & $0.71 \mathrm{mg} / \mathrm{dL}$ \\
Fib & $448 \mathrm{ng} / \mathrm{dL}$ & $\mathrm{NH}{ }_{3}$ & $14 \mu \mathrm{mol} / \mathrm{L}$ \\
D-dimer & $1.5 \mu \mathrm{g} / \mathrm{mL}$ & $\mathrm{BA}$ & $390 \mu \mathrm{mol} / \mathrm{L}$
\end{tabular}

Neut, neutrophil; ALP, alkaline phosphatase; AST, aspartate aminotransferase; ALT, alanine aminotransferasePT, prothrombin time; INR, international normalized ratio; APTT, activated partial thromboplastin time; Fib, fibrinogen.

jaundice or pruritus until the age of 16 . At the age of 16 years and 6 months, jaundice recurred, and the T-Bil value was $3.6 \mathrm{mg} / \mathrm{dL}$ at the time of presentation but rapidly increased to $21.2 \mathrm{mg} / \mathrm{dL}, 3$ days later. Twelve days after presentation, she was referred to our hospital, where laboratory investigations showed T-Bil level of $35.7 \mathrm{mg} / \mathrm{dL}$ (conjugated fraction: $24.6 \mathrm{mg} / \mathrm{dL}$ ), BA of $390 \mu \mathrm{mol} / \mathrm{L}$, alkaline phosphatase of 1,319 U/L; aspartate aminotransferase, alanine aminotransferase, and GGT were within normal limits (Table 2). Serological investigations excluded infectious (hepatitis virus infection), metabolic, and autoimmune causes of acute and chronic liver disease. Computed tomography and magnetic resonance cholangiopancreatography showed normal intra- and extra-hepatic biliary trees. Liver biopsy showed severe intrahepatic bile stasis with bile plugs, and no findings suggestive of chronic hepatitis or cirrhosis. Since jaundice had recurred and no other findings suggested hepatitis, BRIC was suspected, and ATP8B1 and ABCB11 gene analysis was performed after obtaining informed consent. Target next-generation sequencing (NGS) using the hybrid capture method was performed on the genes extracted from the blood of the patient. Genome sequences were analyzed for exons in the protein-coding region and 10 nucleotides in the introns in the border region. The sequences obtained were compared with publicly available human genome reference sequences (GRCh38/hg38) to discover rare sequences, deletion, or insertion of short nucleotide sequences. No variant of $A B C B 11$ was found, but seven variants of $A T P 8 B 1$ were found, five of which are considered genetic polymorphisms in the Japanese population because they had been reported in ToMMo (Tohoku Medical Megabank Organization [jJGVD_8.3kjpn; https://jmorp.megabank.tohoku.ac.jp/ijgvd/]); the remaining two heterozygous missense variants (p.Val997Met and p.Ile516Asn) have never been reported (Table 3). These two variants could be pathological because BRIC is considered an autosomal recessive disorder. For the trio analysis, NGS for the two variants was performed on the parents. As a result, the variant p.Val997Met was confirmed to be of paternal origin and p.Ile516Asn of maternal origin (Fig. 1), and the diagnosis of BRIC1 owing to a compound heterozygous mutation in the ATP8B1 gene was confirmed. The patient was treated with daily dose of urusodeoxycholic acid (300 $\mathrm{mg}$ ) and cholestyramine (4 g). T-Bil levels dropped to $1.1 \mathrm{mg} / \mathrm{dL}$ after 294 days of admission (Fig. 2). 
Table 3. Genetic analysis of the patient

\begin{tabular}{lllllll}
\hline No & Gene & Genotype & Annotation & HGVS.c & HGVS.p & ToMMo \\
\hline 1 & ATP8B1 & Heterozygous & Synonymous variant & c.3744C $>$ A & p.Thr1248= & 0.0555 \\
2 & ATP8B1 & Heterozygous & Synonymous variant & c.3477C $>\mathrm{T}$ & p.Pro1159= & 0.00554 \\
3 & ATP8B1 & Heterozygous & Missense variant & c.2989G $>\mathrm{A}$ & p.Val997Met & No date \\
4 & ATP8B1 & Heterozygous & Missense variant & c.2021T $>\mathrm{C}$ & p.Met674Thr & 0.0544 \\
5 & ATP8B1 & Heterozygous & Missense variant & c.1729A $>\mathrm{G}$ & p.Ile577Val & 0.0544 \\
6 & ATP8B1 & Heterozygous & Missense variant & c.1547T $>\mathrm{A}$ & p.Ile516Asn & No date \\
7 & ATP8B1 & Heterozygous & Missense variant & c.234C $>\mathrm{G}$ & p.His78Gln & 0.0054 \\
\hline
\end{tabular}

There were no variants of $A B C B 11$, but seven variants of $A T P 8 B 1$ were found. Nos. 1 and 2 are synonymous variants, and therefore they could not be a factor in the disease. Nos. 4, 5, and 7 were considered genetic polymorphisms in the Japanese population. Cases 3 and 6 have not been reported previously.

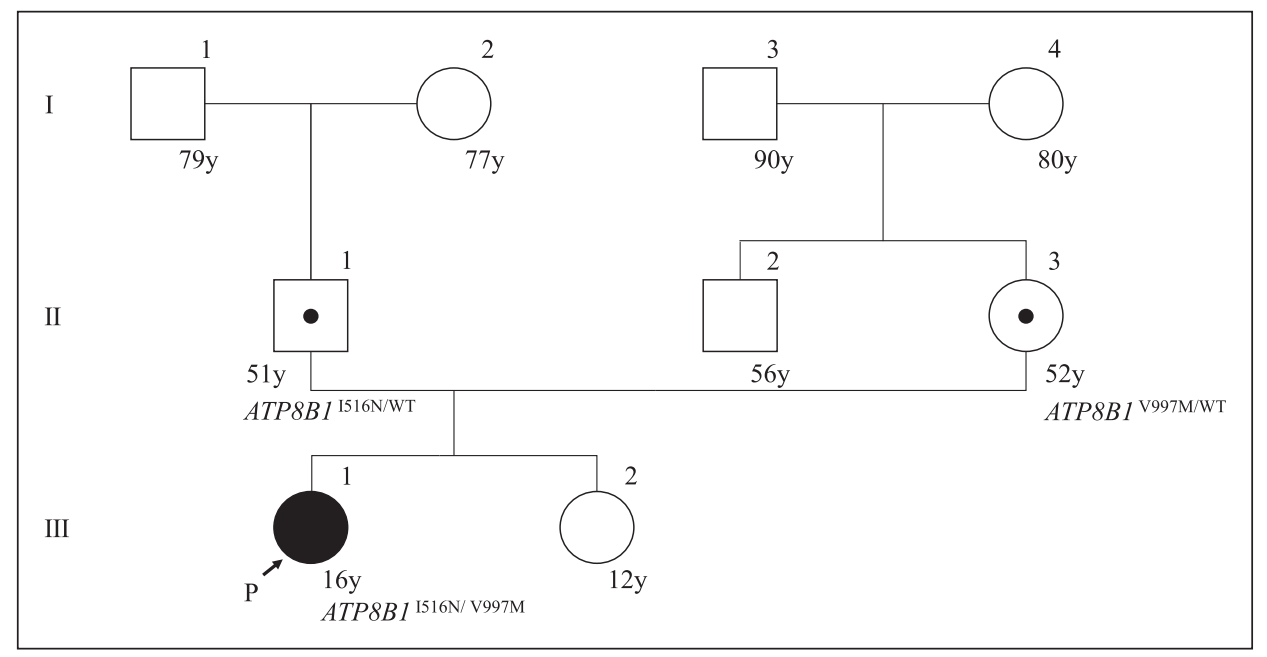

Fig. 1. Pedigree of the ATP8B1 variants (c.Ile516Asn and c.Val997Met) identified in the family of the patient with BRIC1. No one else in her family has developed BRIC1. Patient's father has the heterozygous mutation c.Ile516Asn and mother has the heterozygous mutation c.Val997Met. The compound heterozygous mutation is the cause of BRIC1 in this patient. Genetic analysis was not performed on her sister because she declined consent.

\section{Discussion}

BRIC has been reported several times since it was first reported by Summerskill and Walshe [1], as a disease in which jaundice is recurrent. The widely known and most common diagnostic criteria for BRIC, which are based on clinical symptoms, have been proposed by Luketic and Shiffman [2]. These include the following: at least two episodes of jaundice separated by a symptom-free interval lasting several months to years; laboratory values consistent with intrahepatic cholestasis; normal or minimally elevated GGT; severe pruritus secondary to cholestasis; liver histology demonstrating centrilobular cholestasis; normal intra- and extra-hepatic bile ducts by cholangiography (endoscopic retrograde cholangiopancreatography, magnetic resonance cholangiography); and absence of factors known to be associated with cholestasis (i.e., drugs, pregnancy) [2]. Gene identification is not essential 


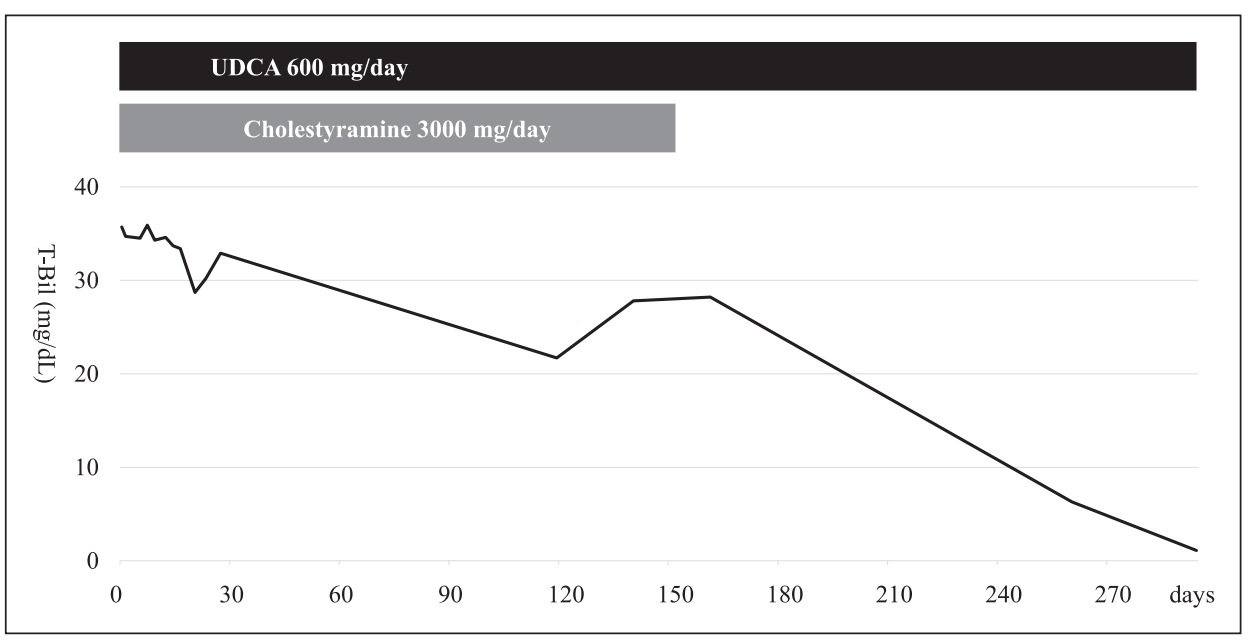

Fig. 2. Clinical course. Cholestyramine was discontinued owing to poor improvement in itching symptoms. At 294 days after the first admission, itching was relieved, and jaundice was reduced.

for diagnosis, but pathological mutation in ATP8B1 has been reported. For instance, Klomp et al. [3] performed a genetic analysis of BRIC families and reported that I661T was identified as a typical mutation. ATP8B1 deficiency causes two forms of familial intrahepatic cholestasis: progressive familial intrahepatic cholestasis type 1 (PFIC1) and BRIC1 (the type found in this case) [4]. PFIC develops in infancy and presents with chronic intrahepatic bile stasis, running a progressive and fatal course. Mutations that cause BRIC1 are thought to have only a partial effect on protein function and expression compared to mutations in PFIC1 [3]. However, several cases of BRIC that subsequently transitioned to a persistent progressive form have been reported. Thus, it is theorized that BRIC and PFIC seem to represent a continuous spectrum of phenotypes of one disease $[5,6]$. These differences result from the difference in the importance of the proteins expressing genetic abnormalities; this has been proven by in vitro and mouse experiments [7]. Therefore, the identification of pathological variants is considered important.

This case met the diagnostic criteria for BRIC and this diagnosis was made. However, since the patient is young (16 years), it may be possible to estimate the severity of the disease (by comparing it with past cases), the risk of the disease developing in her sister and children when she gives birth. To evaluate the risk of developing the disease, a trio analysis should be conducted in consultation with patients and their families. Therefore, it is considered that the two novel nonsense mutations (p.Val997Met and p.Ile516Asn) have high diagnostic value because they have a recessive inheritance pattern. With the development of NGS, genetic analysis has become more common, and the number of case reports of genetic analysis is increasing. BRIC is a rare disease, with many unknowns, including prognosis, and no treatments have been established. It is important to report cases (such as this case) in which variants can be identified, and their course of treatment.

\section{Acknowledgments}

The authors thank all medical workers involved in this study. 


\section{Statement of Ethics}

The authors have no ethical conflicts to disclose. Written informed consent was obtained from both the patient and parents for the publication of this case report and accompanying images because the patient is 16 years old. This study protocol was reviews and the need for approval was waived by the Medical Ethics Committee of the Hiroshima University Hospital, so this case report was exempted from the committee as per its policy.

\section{Conflict of Interest Statement}

The authors declare no conflicts of interest or financial support in association with the study.

\section{Funding Sources}

The authors declare that this study received no funding.

\section{Author Contributions}

R.M., T.K., M.I., M.K., Y.J., Y.S., S.M., S.Y., K.A., K.N., Y.A., Y.K., K.K., S.U., H.F., A.O., T.N., E.M., M.Y., T.H., and H.A. were involved in the interpretation of the data. R.M., T.K., M.I., and T.H. were involved in the conception and the drafting of the manuscript and participated in the revisions. R.M., T.K., M.I., M.K., Y.J., Y.S., S.M., S.Y., K.A., K.N., Y.A., Y.K., K.K., S.U., H.F., A.O., T.N., E.M., M.Y., T.H., and H.A. approved the final version of the manuscript.

\section{Data Availability Statement}

Data sharing is not applicable to this study as no datasets were generated or analyzed for writing this study. All significant data generated or analyzed during this study are included in this article. Further inquiries can be directed to the corresponding author.

\section{References}

1 Summerskill WH, Walshe JM. Benign recurrent intrahepatic "obstructive” jaundice. Lancet. 1959 0ct;2(7105): 686-90.

2 Luketic VA, Shiffman ML. Benign recurrent intrahepatic cholestasis. Clin Liver Disvii. 2004 Feb;8(1):133-49, vii.

3 Klomp LW, Vargas JC, van Mil SW, Pawlikowska L, Strautnieks SS, van Eijk MJ, et al. Characterization of mutations in ATP8B1 associated with hereditary cholestasis. Hepatology. 2004 Jul;40(1):27-38.

4 Bull LN, van Eijk MJ, Pawlikowska L, DeYoung JA, Juijn JA, Liao M, et al. A gene encoding a P-type ATPase mutated in two forms of hereditary cholestasis. Nat Genet. 1998 Mar;18(3):219-24.

5 Sticova E, Jirsa M, Pawłowska J. New insights in genetic cholestasis: from molecular mechanisms to clinical implications. Can J Gastroenterol Hepatol. 2018;2018:2313675.

6 van Ooteghem NA, Klomp LW, van Berge-Henegouwen GP, Houwen RH. Benign recurrent intrahepatic cholestasis progressing to progressive familial intrahepatic cholestasis: low GGT cholestasis is a clinical continuum. J Hepatol. 2002 Feb;36(3):439-43.

7 Folmer DE, van der Mark VA, Ho-Mok KS, Oude Elferink RP, Paulusma CC. Differential effects of progressive familial intrahepatic cholestasis type 1 and benign recurrent intrahepatic cholestasis type 1 mutations on canalicular localization of ATP8B1. Hepatology. 2009 Nov;50(5):1597-605. 